\title{
Massive binaries as the source of abundance anomalies in globular clusters
}

\author{
S. E. de Mink ${ }^{1}$, O. R. Pols ${ }^{1}$, N. Langer ${ }^{2,1}$, and R. G. Izzard ${ }^{3}$ \\ 1 Astronomical Institute, Utrecht University, PO Box 80000, 3508 TA Utrecht, The Netherlands \\ e-mail: [S.E.deMink;0.R.Pols]@uu.nl \\ 2 Argelander-Institut für Astronomie der Universität Bonn, Auf dem Hügel 71, 53121 Bonn, Germany \\ e-mail: nlanger@astro.uni-bonn.de \\ 3 Université Libre de Bruxelles, Boulevard du Triomphe, 1050 Brussels, Belgium \\ e-mail: Robert.Izzard@ulb.ac.be
}

Received 29 August 2009 / Accepted 1 October 2009

\section{ABSTRACT}

\begin{abstract}
Abundance anomalies observed in globular cluster stars indicate pollution with material processed by hydrogen burning. Two main sources have been suggested: asymptotic giant branch (AGB) stars and massive stars rotating near the break-up limit (spin stars). We propose massive binaries as an alternative source of processed material. We compute the evolution of a $20 M_{\odot}$ star in a close binary considering the effects of non conservative mass and angular momentum transfer and of rotation and tidal interaction to demonstrate the principle. We find that this system sheds about $10 M_{\odot}$ of material, nearly the entire envelope of the primary star. The ejecta are enriched in $\mathrm{He}, \mathrm{N}, \mathrm{Na}$, and $\mathrm{Al}$ and depleted in $\mathrm{C}$ and $\mathrm{O}$, similar to the abundance patterns observed in gobular cluster stars. However, $\mathrm{Mg}$ is not significantly depleted in the ejecta of this model. In contrast to the fast, radiatively driven winds of massive stars, this material is typically ejected with low velocity. We expect that it remains inside the potential well of a globular cluster and becomes available for the formation or pollution of a second generation of stars. We estimate that the amount of processed low-velocity material ejected by massive binaries is greater than the contribution of AGB stars and spin stars combined, assuming that the majority of massive stars in a proto-globular cluster interact with a companion and return their envelope to the interstellar medium. If we take the possible contribution of intermediate mass stars in binaries into account and assume that the ejecta are diluted with an equal amount of unprocessed material, we find that this scenario can potentially provide enough material to form a second generation of low-mass stars, which is as numerous as the first generation of low-mass stars, without the need to make commonly adopted assumptions, such as preferential loss of the first generation of stars, external pollution of the cluster, or an anomalous initial mass function.
\end{abstract}

Key words. stars: abundances - stars: binaries: close - Galaxy: globular clusters: general - ISM: general

\section{Introduction}

For a long time star clusters have been considered as idealized single-age, chemically homogeneous stellar populations. However, it has recently become clear that many clusters show multiple main sequences and sub giant branches and extended horizontal branches (e.g. Piotto et al. 2007), implying the existence of multiple populations within one cluster.In addition, large star-to-star abundance variations are found for light elements such as $\mathrm{C}, \mathrm{N}, \mathrm{O}, \mathrm{Na}$, and $\mathrm{Al}$, while the composition of heavier elements (Fe-group and $\alpha$-elements) seems to be constant. Field stars with the same metallicity do not exhibit these abundance patterns (for a review see Gratton et al. 2004). These chemical variations have been interpreted as originating from the presence of both a "normal" stellar population, exhibiting abundances similar to field stars of the same metallicity and a second population of stars formed out of material processed by hydrogen burning via the CNO-cycle and by the $\mathrm{NeNa}$ and $\mathrm{MgAl}$ chains (e.g. Prantzos et al. 2007). According to Carretta et al. (2009), 50-70\% of the stars in gloular clusters belong to the second population.

Two sources of processed ejecta have been proposed: the slow winds of massive $A G B$ stars, which enrich their convective envelopes with H-burning products (Ventura et al. 2001;
D'Antona et al. 2002; Denissenkov \& Herwig 2003) and fastrotating massive stars (we refer to these as spin stars), which are believed to expel processed material centrifugally when they reach break-up rotation (Prantzos \& Charbonnel 2006; Decressin et al. 2007b).In this scenario a first generation of stars is formed out of pristine material. Their low-velocity ejecta are trapped inside the potential well of the cluster and provide the material for the formation of a second generation of stars. Although both proposed sources are promising, matching the observed abundance patterns and providing enough ejecta for the formation of a second generation that outnumbers the first generation have proven to be two major challenges. In this Letter we propose massive binaries as a candidate for the internal pollution of globular clusters.

\section{Binaries as sources of enrichment}

Interacting binaries can shed large amounts of material processed by hydrogen burning into their surroundings. A clear example is the well-studied system RY Scuti. It is undergoing rapid mass transfer from a $7 M_{\odot}$ supergiant to its $30 M_{\odot}$ companion. Mass is lost from the system via the outer Lagrangian points into a circumbinary disk and a wider double toroidal nebula. The nebula shows signatures of CNO processing: it is enriched in 
helium and nitrogen and depleted in oxygen and carbon (Smith et al. 2002; Grundstrom et al. 2007). In contrast to the highvelocity radiatively driven winds of massive stars, these ejecta have low velocities. Smith et al. (2001) measure expansion velocities ranging from 30 to $70 \mathrm{~km} \mathrm{~s}^{-1}$ in the nebula of RY Scuti, which are lower than the present-day escape velocity of massive globular clusters. Furthermore, the nebula shows evidence of clumps (Smith et al. 2002) and dust in the outer parts (Gehrz et al. 2001), which may serve as seeds for forming a second generation of low-mass proto stars.

Evidence of severe mass loss from interacting binaries comes from a wide variety of observed interacting and postinteraction systems. This appears to be a common phenomenon for many interacting binaries. Various authors have inferred highly non conservative evolution for Algols, systems that are currently undergoing stable mass transfer (e.g. Refsdal et al. 1974; de Greve \& Linnell 1994; Figueiredo et al. 1994; van Rensbergen et al. 2006; De Mink et al. 2007). Most notable are short-period binaries containing a compact object, e.g. cataclysmic variables, X-ray binaries, binary radio pulsars and double white dwarf systems. Their formation requires a phase of severe mass and angular momentum loss by ejection of a common envelope. Direct evidence of this type of evolution comes from planetary nebulae with close binary nuclei, which appear to have recently emerged from the common-envelope phase (for a review see Iben \& Livio 1993).

Theoretical considerations support the idea that most interacting binaries shed large amounts of mass. Three-dimensional hydrodynamical simulations of the mass transfer stream and accretion disk of the interacting binary $\beta$ Lyrae predict that $50 \%$ of the transferred mass is lost (Bisikalo et al. 2000; Nazarenko \& Glazunova 2006). In addition, Ulrich \& Burger (1976) showed that the accreting star is driven out of thermal equilibrium and expands. This can lead to contact and to strong mass and angular momentum loss from the system (Flannery \& Ulrich 1977). Furthermore, Packet (1981) noted that the accreting star reaches break-up rotation after gaining only a few percent of its own mass. Rapid rotation is found for many accreting stars in Algols (Barai et al. 2004) and this mechanism has been proposed to explain the formation of Be-X-ray binaries (e.g. Pols et al. 1991). In principle, tides can counteract the effect of spin-up by mass transfer in close binaries. Petrovic et al. (2005a) have computed detailed binary evolution models taking these effects into account. They find that massive binaries with initial periods as short as 3-6 days lose 70-80\% of the transferred mass on average. For wider and more massive systems, they expect even less conservative mass transfer, such that nearly the entire envelope of the primary is returned to the interstellar medium.

\section{Composition of the ejecta}

To investigate the yields of a typical massive binary, we employ a state-of-the-art binary evolution code described by Petrovic et al. (2005b) and Yoon et al. (2006). The effect of mass and angular momentum loss on the binary orbit is computed according to Podsiadlowski et al. (1992), with the specific angular momentum of the wind calculated according to Brookshaw \& Tavani (1993). Mass transfer is modeled following Ritter (1988). Tidal interaction is modeled as described in Detmers et al. (2008). Non conservative mass transfer is modeled self-consistently: it results from the interplay between spin-up by mass transfer, tidal interaction, and rotationally enhanced mass loss. To follow the nucleosynthesis up to the advanced stages of hydrogen burning, we updated the reaction rates to the NACRE 99 compilation (Angulo et al. 1999). As initial composition we assumed an $\alpha$-enhanced mixture with a metallicity of $Z=5 \times 10^{-4}$, following Decressin et al. (2007b). We assumed masses of 20 and $15 M_{\odot}$ for the two stars, an orbital period of 12 days and initial rotation rates synchronized with the orbital revolution. Because of these low initial rotation rates, the effect of rotationally induced mixing on the low-velocity ejecta is negligible. We follow the evolution from the onset of hydrogen burning until central carbon burning.

Shortly after hydrogen exhaustion in the center, the primary expands and starts to transfer mass to its companion. Initially the secondary star efficiently accretes all the transferred mass and associated angular momentum and spins up. After accreting about $1.5 M_{\odot}$ it approaches critical rotation. From this moment on, the majority of the transferred mass is ejected from the system.

The transition from conservative to non conservative mass transfer, when the accreting star reaches critical rotation, provides an interesting selection mechanism. Initially, when the outermost unprocessed layers are transferred, the companion star efficiently accretes all the material. By the time deeper layers of the donor star, which do show signatures of nuclear processing, are exposed, mass is ejected from the system.

After transferring nearly its entire envelope, the donor contracts, ignites helium, and becomes a Wolf-Rayet star. In the meantime the secondary spins down because of angular momentum loss in its rotationally enhanced wind. A second phase of mass transfer sets in when the Wolf-Rayet star expands during He-shell burning. This time about $1 M_{\odot}$ is transferred, which is initially accreted by the secondary but ejected shortly afterwards by its rotationally enhanced wind. Our computation ends after the primary star ignites carbon and fills its Roche lobe a third time. Soon after, it will explode as a type Ib/c supernova.

Figure 1 shows the composition of the material ejected from the system during the two phases of mass transfer. The first $2 M_{\odot}$ are relatively unprocessed and resemble the pristine composition, except for the depletion of fragile elements such as lithium (not plotted). The next $2 M_{\odot}$ of ejected material are processed by $\mathrm{CN}$ cycling (nitrogen being enhanced up to a factor five), followed by about $4 M_{\odot}$ showing He enrichment and the O-Na anti-correlation. After the ejection of $8 M_{\odot}$, a sudden change in slope is visible for all elements except carbon. The layers of the donor star, which are exposed at this moment, were part of the convection zone above the $\mathrm{H}$ burning shell. Here, the temperatures were high enough for proton captures onto ${ }^{25} \mathrm{Mg}$ and ${ }^{26} \mathrm{Mg}$, leading to an increase in the aluminum abundance by a factor two. Between the first and second mass transfer phases, the primary star loses mass in the form of a fast WolfRayet wind, and even deeper layers of the primary are exposed. Because the these high-velocity winds are likely to escape from the cluster, we have exclude the mass ejected during this phase in Fig. 1.

The average composition of the ejecta are comparable to the yields of a $60 M_{\odot}$ spin star model computed by Decressin et al. (2007b) by adopting the same set of reaction rates. For example, we find an average helium mass fraction of 0.30 and an enhancement in sodium by 1.0 dex compared to 0.32 and 1.3 dex, respectively, in the $60 M_{\odot}$ spin star. The sum of carbon, nitrogen, and oxygen is constant within a few percent, consistent with observations (see references in Decressin et al. 2009). However, the temperatures are not high enough for efficient proton captures onto the most abundant isotope of magnesium, ${ }^{24} \mathrm{Mg}$, adopting the recommended reaction rates. The onset of this reaction is required to explain the full range of aluminum abundances 

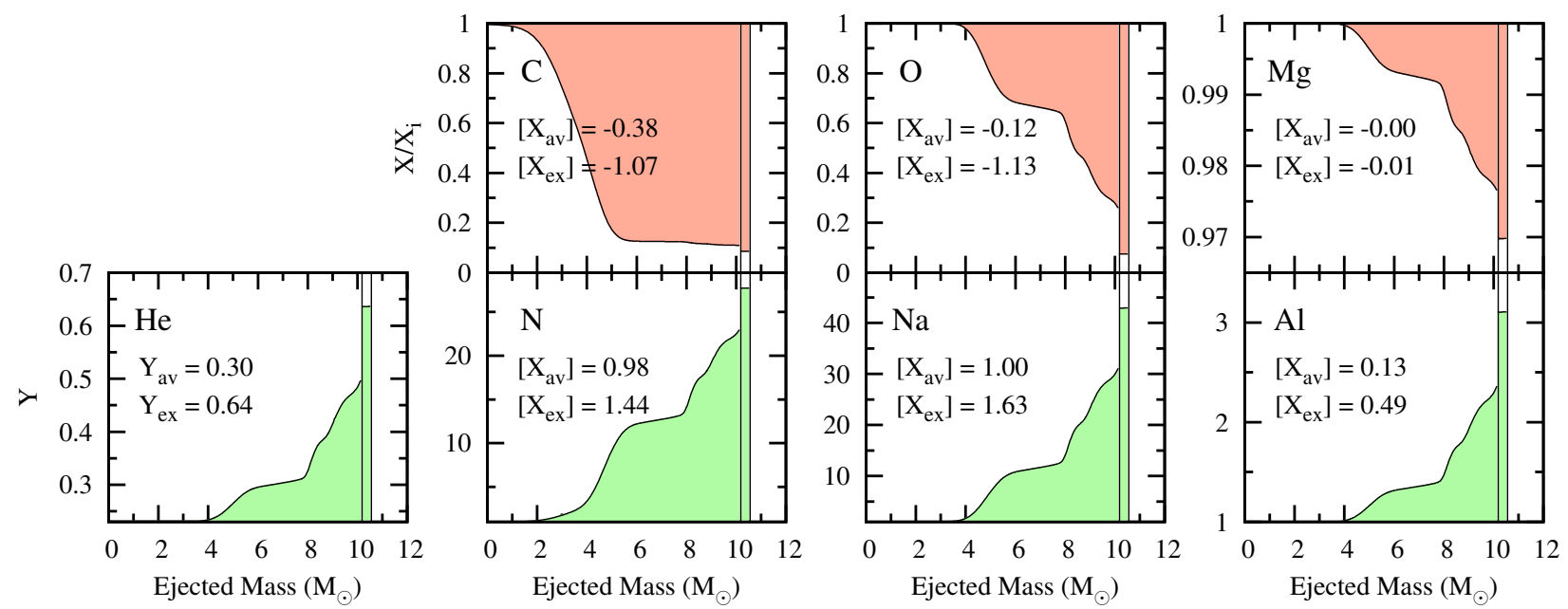

Fig. 1. Composition of the slow ejecta of the modeled binary system (Sect. 3) as a function of the ejected amount of mass. The mass fraction $X$ of the main stable isotope of each element is given relative to the initial mass fraction $X_{\mathrm{i}}$, except for $\mathrm{Mg}$ where we added ${ }^{24} \mathrm{Mg}{ }^{25} \mathrm{Mg}$ and ${ }^{26} \mathrm{Mg}$. The average $X_{\text {av }}$ and the most extreme mass fraction $X_{\text {ex }}$ are given in each panel on a logarithmic scale: $[X] \equiv \log _{10}\left(X / X_{\mathrm{i}}\right)$. For helium we show the absolute mass fraction $Y$ instead. Mass ejected during the first and second mass transfer phases is separated by a thin vertical line.

observed in some globular clusters. We expect that considering more massive or wider binaries (in which mass transfer starts in a later evolution stage) might alleviate this problem.

In shorter-period binaries, tides can counteract the effect of spin-up: more mass is accreted before the accreting star reaches break-up rotation and less mass is lost from the system. However, the ejecta originate from the last exposed (deepest) layers of the donor star and will therefore show more strongly pronounced anti-correlations. For initially wider systems, we expect that nearly the complete envelope is ejected. In addition stars may have considerable rotation rates at birth. For these stars, mixing induced by rotation can lead to processing of the whole envelope (e.g. Yoon et al. 2006; Decressin et al. 2007b).

Besides the effects discussed above, the (remainders of the) binary can still shed large amounts of H-processed material at low velocities in various ways. The companion star is now rapidly rotating and may reach break-up rotation again towards the end of its evolution as proposed in the spin star scenario. Processed mass can be ejected during a phase of reverse mass transfer from the secondary onto the compact object, if the system remains bound after the supernova explosion of the primary star.

\section{Mass budget}

One of the main challenges for the two previously proposed sources of pollution, massive AGB and spin stars, is to provide the large amount of ejecta needed to create a second population that is larger than the first population. The population of low-mass stars $\left(0.1-0.8 M_{\odot}\right)$, which can still be observed today, represents $38 \%$ of the stellar mass initially present in the cluster assuming a standard Kroupa (2001) initial mass function (IMF) between 0.1-120 $M_{\odot}$, see Fig. 2. The ejecta of AGB stars with initial masses between 4 and $9 M_{\odot}$ represent up to $8.9 \%$ of the initial stellar mass (assuming an initial-final mass relation by Ciotti et al. 1991). For spin stars this fraction is $3.4 \%$, if one assumes that every massive star is single and born with a rotational velocity high enough to reach break-up rotation (using models by Decressin et al. 2007b). There are not enough of these ejecta to create a second generation which is equally numerous as the first generation, even when we assume that the second

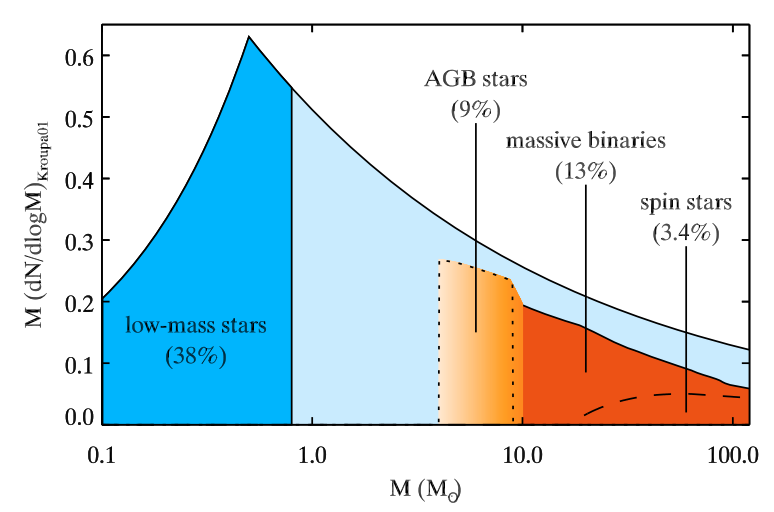

Fig. 2. Mass-weighted Kroupa (2001) IMF as a function of stellar mass. The surface areas indicate the mass contained in the first generation of long-lived low-mass stars (dark blue), the ejecta of AGB stars (dotted line), spin stars, i.e. fast-rotating massive stars (dashed line), and massive (red) and intermediate-mass (orange) binaries. Percentages indicate the fraction of mass relative to the total mass contained in stars of the first generation. See Sect. 4 for details.

generation consists only of low-mass stars and that star formation is very efficient, see Fig. 2.

Two rather extreme solutions have been proposed. (1) The IMF was highly anomalous, favoring the formation of the polluting stars with respect to the long-lived low-mass stars that we observe today. Even though we have no direct constraints on the IMF of globular clusters, Kroupa (2002) finds that the IMF is remarkably uniform in stellar populations with very different properties; (2) Clusters were initially at least 10-20 times more massive and they have primarily lost low-mass stars from the first generation as a result of the dynamical evolution and tidal stripping of the cluster (Decressin et al. 2007a, 2008; D'Ercole et al. 2008). In this section we investigate to what extent the ejecta of massive binaries can alleviate this conundrum.

Even though the current fraction of detected binaries in globular clusters is not high (e.g. Davis et al. 2008), this is not necessarily the case for the high-mass stars originally present in the cluster. Sana et al. (2008) and Mason et al. (2009) find a minimum binary fraction of $60-75 \%$ for $\mathrm{O}$ stars associated with clusters or $\mathrm{OB}$ associations. In globular clusters, these 
fractions may even be higher. In this environment, close binaries can be created and tightened during and after the star formation process, for example by the dissipative interaction with gas (e.g. Bonnell \& Bate 2005) and by three-body interactions, such as the Kozai mechanism in combination with tidal friction (Fabrycky \& Tremaine 2007). With massive stars preferentially residing in the dense core of the cluster, where the dynamical encounters are most frequent, it is not unreasonable to assume that the large majority of massive stars interact by mass exchange.

Let us assume that every massive star is a member of an interacting binary. In Sects. 2 and 3, we argued that nearly the entire envelope of the donor is returned to the ISM. For simplicity, we neglect the contribution of the secondary star after it has been spun up by mass transfer or during a possible phase of reverse mass transfer, and we assume that the entire envelope of the primary becomes available for star formation. We assume heliumcore masses as in Prantzos \& Charbonnel (2006) for stars more massive than $10 M_{\odot}$. Under these assumptions, the slow ejecta of massive binaries represent $13 \%$ of the mass originally present in stars: more than the ejecta of AGB and spin stars combined.

Measurements of lithium suggest that the ejecta of the first generation are diluted with pristine gas (Pasquini et al. 2005). Together with an equal amount of pristine gas, the ejecta of binaries with donors more massive than $10 M_{\odot}$ would represent $26 \%$ of the initial cluster mass (compared to $38 \%$ contained in the first generation of low-mass stars). The adopted lower mass limit for our binary scenario is rather arbitrary. If we take the potential contribution of intermediate mass stars $\left(4-10 M_{\odot}\right)$ into account according to this scenario, there would be enough ejecta to form a second population of chemically peculiar stars that outnumbers the first generation of normal stars. The assumptions in this scenario can be relaxed if the evaporation of stars from the cluster primarily affects the first stellar generation, as suggested by D'Ercole et al. (2008) and Decressin et al. (2008).

\section{Conclusions}

We propose massive binaries as a source for the internal pollution of globular clusters. The majority of massive stars are expected to be members of interacting binary systems. These return most of the envelope of their primary star to the interstellar medium during non conservative mass transfer. We show that there may be more polluted material ejected by binaries than by the two previously suggested sources: massive AGB stars and the slow winds of fast-rotating massive stars. After dilution with pristine material, as lithium observations suggest, binaries could return enough material to form a chemically enriched second generation that is as numerous as the first generation of lowmass stars, without the need to assume a highly anomalous IMF, external pollution of the cluster or a significant loss of stars from the unenriched first generation.

In addition to providing a new source of slowly-ejected enriched material, binary interaction also affects the previously proposed scenarios. Binary mass transfer naturally produces a large number of fast-rotating massive stars that may enrich their surroundings even more. Binary interaction will also affect the yields of intermediate-mass stars. Premature ejection of the envelope in 4-9 $M_{\odot}$ stars will result in ejecta with less pronounced anti-correlations, as suggested in the AGB scenario. On the other hand, we expect that binary-induced mass loss may also prevent the dredge-up of helium-burning products.
For a detailed comparison of the chemical predictions of this scenario, binary models for a range of masses and orbital periods are needed and population synthesis models are essential to fullly evaluate the mass budget of the different sources. Finally, some peculiar feature, such as the apparent presence of distinct, chemically homogeneous subpopulations in $\omega$ Cen and NGC 2808 (e.g. Renzini 2008) deserves further attention.

Acknowledgements. We thank T. Decressin, E. Glebbeek, A. Karakas, C. Charbonnel, B. van Veelen, M. Cantiello, and the referee F. D'Antona for useful discussions.

\section{References}

Angulo, C., Arnould, M., Rayet, M., et al. 1999, Nucl. Phys. A, 656, 3 Barai, P., Gies, D. R., Choi, E., et al. 2004, ApJ, 608, 989

Bisikalo, D. V., Harmanec, P., Boyarchuk, A. A., Kuznetsov, O. A., \& Hadrava, P. 2000, A\&A, 353, 1009

Bonnell, I. A., \& Bate, M. R. 2005, MNRAS, 362, 915

Brookshaw, L., \& Tavani, M. 1993, ApJ, 410, 719

Carretta, E., Bragaglia, A., Gratton, R. G., et al. 2009, A\&A, in press

Ciotti, L., D'Ercole, A., Pellegrini, S., \& Renzini, A. 1991, ApJ, 376, 380

D’Antona, F., Caloi, V., Montalbán, J., Ventura, P., \& Gratton, R. 2002, A\&A, 395, 69

Davis, D. S., Richer, H. B., Anderson, J., et al. 2008, AJ, 135, 2155

de Greve, J. P., \& Linnell, A. P. 1994, A\&A, 291, 786

De Mink, S. E., Pols, O. R., \& Hilditch, R. W. 2007, A\&A, 467, 1181

Decressin, T., Charbonnel, C., \& Meynet, G. 2007a, A\&A, 475, 859

Decressin, T., Meynet, G., Charbonnel, C., Prantzos, N., \& Ekström, S. 2007b, A\&A, 464, 1029

Decressin, T., Baumgardt, H., \& Kroupa, P. 2008, A\&A, 492, 101

Decressin, T., Charbonnel, C., Siess, L., et al. 2009, A\&A, 505, 727

Denissenkov, P. A., \& Herwig, F. 2003, ApJ, 590, L99

D’Ercole, A., Vesperini, E., D’Antona, F., McMillan, S. L. W., \& Recchi, S. 2008, MNRAS, 391, 825

Detmers, R. G., Langer, N., Podsiadlowski, P., \& Izzard, R. G. 2008, A\&A, 484, 831

Fabrycky, D., \& Tremaine, S. 2007, ApJ, 669, 1298

Figueiredo, J., de Greve, J. P., \& Hilditch, R. W. 1994, A\&A, 283, 144

Flannery, B. P., \& Ulrich, R. K. 1977, ApJ, 212, 533

Gehrz, R. D., Smith, N., Jones, B., Puetter, R., \& Yahil, A. 2001, ApJ, 559, 395 Gratton, R., Sneden, C., \& Carretta, E. 2004, ARA\&A, 42, 385

Grundstrom, E. D., Gies, D. R., Hillwig, T. C., et al. 2007, ApJ, 667, 505

Iben, I. J., \& Livio, M. 1993, Pub. Astron. Soc. Pacific, 105, 1373

Kroupa, P. 2001, MNRAS, 322, 231

Kroupa, P. 2002, Science, 295, 82

Mason, B. D., Hartkopf, W. I., Gies, D. R., Henry, T. J., \& Helsel, J. W. 2009, AJ, 137, 3358

Nazarenko, V. V., \& Glazunova, L. V. 2006, Astron. Rep., 50, 380

Packet, W. 1981, A\&A, 102, 17

Pasquini, L., Bonifacio, P., Molaro, P., et al. 2005, A\&A, 441, 549

Petrovic, J., Langer, N., \& van der Hucht, K. A. 2005a, A\&A, 435, 1013

Petrovic, J., Langer, N., Yoon, S.-C., \& Heger, A. 2005b, A\&A, 435, 247

Piotto, G., Bedin, L. R., Anderson, J., et al. 2007, ApJ, 661, L53

Podsiadlowski, P., Joss, P. C., \& Hsu, J. J. L. 1992, ApJ, 391, 246

Pols, O. R., Cote, J., Waters, L. B. F. M., \& Heise, J. 1991, A\&A, 241, 419

Prantzos, N., \& Charbonnel, C. 2006, A\&A, 458, 135

Prantzos, N., Charbonnel, C., \& Iliadis, C. 2007, A\&A, 470, 179

Refsdal, S., Roth, M. L., \& Weigert, A. 1974, A\&A, 36, 113

Renzini, A. 2008, MNRAS, 391, 354

Ritter, H. 1988, A\&A, 202, 93

Sana, H., Gosset, E., Nazé, Y., Rauw, G., \& Linder, N. 2008, MNRAS, 386, 447 Smith, N., Gehrz, R. D., \& Goss, W. M. 2001, AJ, 122, 2700

Smith, N., Gehrz, R. D., Stahl, O., Balick, B., \& Kaufer, A. 2002, ApJ, 578, 464

Ulrich, R. K., \& Burger, H. L. 1976, ApJ, 206, 509

van Rensbergen, W., de Loore, C., \& Jansen, K. 2006, A\&A, 446, 1071

Ventura, P., D’Antona, F., Mazzitelli, I., \& Gratton, R. 2001, ApJ, 550, L65

Yoon, S.-C., Langer, N., \& Norman, C. 2006, A\&A, 460, 199 